\title{
Practical Wide Frequency Approach for Calculating Eddy Current Losses in Transformer Windings
}

\author{
Alex Van den Bossche*, Vencislav Cekov Valchev, Stefan Todorov Barudov \\ * Ghent University, Electrical Energy, Systems and Automation Department, Ghent, B-9000, Belgium \\ Technical University of Varna, Studenska Str.1, Varna 9010, Bulgaria \\ alex.vandenbossche@UGent.be, vencivalchev@hotmail.com
}

\begin{abstract}
A practical method for calculating eddy current losses in transformer windings is reported. The method improves the classical loss presentation by introducing a loss coefficient, called eddy current factor $k_{c}$. In this paper, eddy current losses in round conductors are discussed. A graphical approximation of $\boldsymbol{k}_{c}$ as a function of wire diameter, frequency, layer number, copper packing factors in the direction parallel and perpendicular to the layer is provided. The graphs are obtained by analytical expressions compared with FEM simulations. To unify the approach for different cases, a reference diameter, apparent and equivalent frequency are defined. A few short examples for applying the method in transformer design are given. The method is applicable for a variety of transformers with different frequencies, wire diameters and conductor fittings.

The proposed method is verified by designing several transformers. As an example, a $2.5 \mathrm{~kW}$ transformer is fully described. The experiments show good matching with the calculations.
\end{abstract}

Key words: Magnetic components, Power transformers, Eddy current losses

\section{INTRODUCTION}

Eddy current effects are quite important in inductors and transformers for power electronics. Eddy current losses in round wires, including skin and proximity effects in transformers are discussed by Dowell [1] and many new papers [2], [3], related to some extent to Dowell's interpretation and results. The 2-D analytical approximations are mainly focussed on uniform transverse fields (proximity losses) and also on the own field of a wire (skin-effect losses) [4], [5], [6]. The limitation of the conversion of round wires into rectangular ones is improved in [7], [8]. The optimal design and minimization of losses in Litz wire windings are provided in [9]. Full numerical methods can need up to 1 million of elements easily [10]. This affects calculation time and accuracy.

A conclusion can be made that on one hand, a lot of the design approaches are a compromise of the accuracy in eddy current losses estimation, and on the other hand, the calculation time and complicity.

In this paper a time-saving high accuracy method for eddy current loss calculation in transformer windings is introduced. The proposed approach is applicable for transformers in wide range of designs using round wires.

\section{PROPOSED WIDE FREQUENCY APPROACH FOR CALCULATING EDDY CURRENT LOSSES IN TRANSFORMER WINDINGS}

First, low frequency (LF) approximation is considered [11]. The LF approximation is the starting point of the proposed approach.

The LF approximation is applicable when the eddy currents, induced in the winding do not considerably change the applied field inside the conductor. The LF approximation is valid up $d \leq 1.6 \delta[11],(d$ is the wire diameter and $\delta$ is the penetration depth $\delta=\sqrt{\frac{2 \rho_{\mathrm{c}}}{\omega \mu}}, \omega=2 \pi f$ is the frequency of the applied magnetic field; $\mu$ is the permeability of the material (for copper $\left.\mu \cong \mu_{o}\right), \mu_{o}$ is the permeability of free space; $\mu_{\mathrm{o}}=1.25664 \times 10^{-6} ; \rho_{\mathrm{c}}$ is the electrical resistivity of the conducting material (copper), for copper $\rho_{\mathrm{c}}=23 \times 10^{-9} \Omega \mathrm{m}$ at $100^{\circ} \mathrm{C} ; \rho_{\mathrm{c}}=17 \times 10^{-9} \Omega \mathrm{m}$ at $25^{\circ} \mathrm{C}$.

The major part of the eddy current losses in LF approximation for round wires can be explained by the presence of a transverse magnetic field, which acts like an induction heating to the wire. To express the eddy current losses $P_{c u, e d d y}(t)$, the following equation can be used:

$$
P_{c u, e d d y}(t)=\frac{\pi l_{w} d^{4}}{64 \rho_{\mathrm{c}}}\left(\frac{d B}{d t}\right)^{2}
$$

where:

$d$ is the practical diameter of the copper wire of the considered winding;

$B$ is the induction, assumed perpendicular to the wire axis; $l_{w}$ is the wire length of the winding

The equation (1) is quite general and applicable even for the cases with more complicated field distribution, e.g. for nonsinusoidal waveforms, [3].

\section{A. Eddy current losses factor $k_{c}$}

To extend the validity of (1) for larger diameters, and a wide frequency range, the loss factor $k_{c}$ is introduced. The factor $k_{c}$ represents the ratio between the eddy current losses compared 
to the losses in the ohmic resistance of the winding of the magnetic component.

Using the above introduced loss factor $k_{c}$, the eddy current losses are given by the following equation

$$
P_{e d d y}=\left(R_{0} I_{a c}^{2}\right) k_{c}
$$

where:

$k_{c}$ is the loss factor, depends on the operating frequency $f$, the wire diameter $d$ and the distance between the conductors, presented by the parameter $\eta$ and the distance between the layers presented by the parameter $\lambda$; $I_{a c}$ is the AC current component;

$R_{0}$ is the ohmic resistance of the winding.

The copper fill factor in the direction of the layer $\eta$ can be defined as $\eta=n d / w$ ), where $d$ is the wire diameter; $n$ in the number of turns in a layer; $w$ is the winding width.

The copper fill factor in the direction perpendicular to the layer $\lambda$ can be defined as $\lambda=m_{E} d / h$, where $h$ is the window height (for $m_{E}$ see below).

To allow an easy use of $k_{c}$, we provide two sets of graphs shown in Fig. 1 and Fig.2, giving the additional coefficient $k_{t f}$. For the transformers the value of the coefficient $k_{c}$ is:

$$
k_{c} \approx m_{E}^{2} k_{t f}
$$

where the value of $k_{t f}$ is found using Fig.1 and Fig.2.

The parameter $m_{E}$ (equivalent number of layers) is defined as the number of layers going from no field to the maximum field.

For p parallel wires a number of wires is present in the same layer, which will become an equivalent number of wires in a layer: $m_{E}=m$.

For Litz wire one cannot count exactly the number of individual wires in a layer. We distribute the equivalent turns in both directions and then we have: $m_{E}=m \sqrt{p}$, where $p$ is the number of paralleled strands.

For interleaved windings in transformers (and no parallel wires) $m_{E}=m / K$, where $K$ is the field symmetry factor [11]. This presentation is also necessary for so called 'half layers'.

It is not recommended to use partially filled layers in transformer designs. If anyhow partially filled layers are used, in the proposed method, the wires should be equally spread. The effect of the partially filled layers is reduced at high values of $m_{E}$.

The graphs are obtained by approximating the exact solution for eddy current losses for different cases, found by using software package MathCad [11].

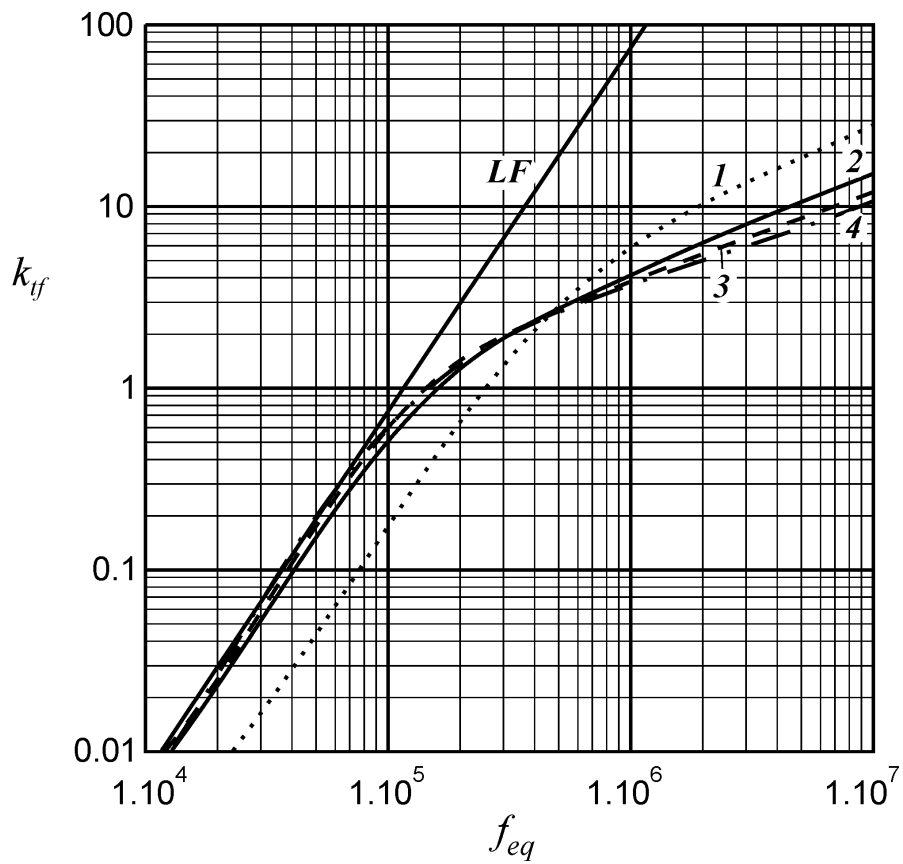

Fig. 1 Transformer cases, typical transformer factor $k_{t f}$ for $\mathrm{d}=0.5 \mathrm{~mm}, \eta=0.9$, $\rho=23 \times 10^{-9}$ and $\left.\lambda=0.5,1\right)$ dotted line: half layer, $\left.m_{E}=0.5 ; 2\right)$ solid line: single layer, $m_{E}=1 ; 3$ ) dashed: two layers, $\left.m_{E}=2 ; 4\right)$ dash-dot: three or more layers, $m_{E}>2$. $L F-$ low frequency approximation.

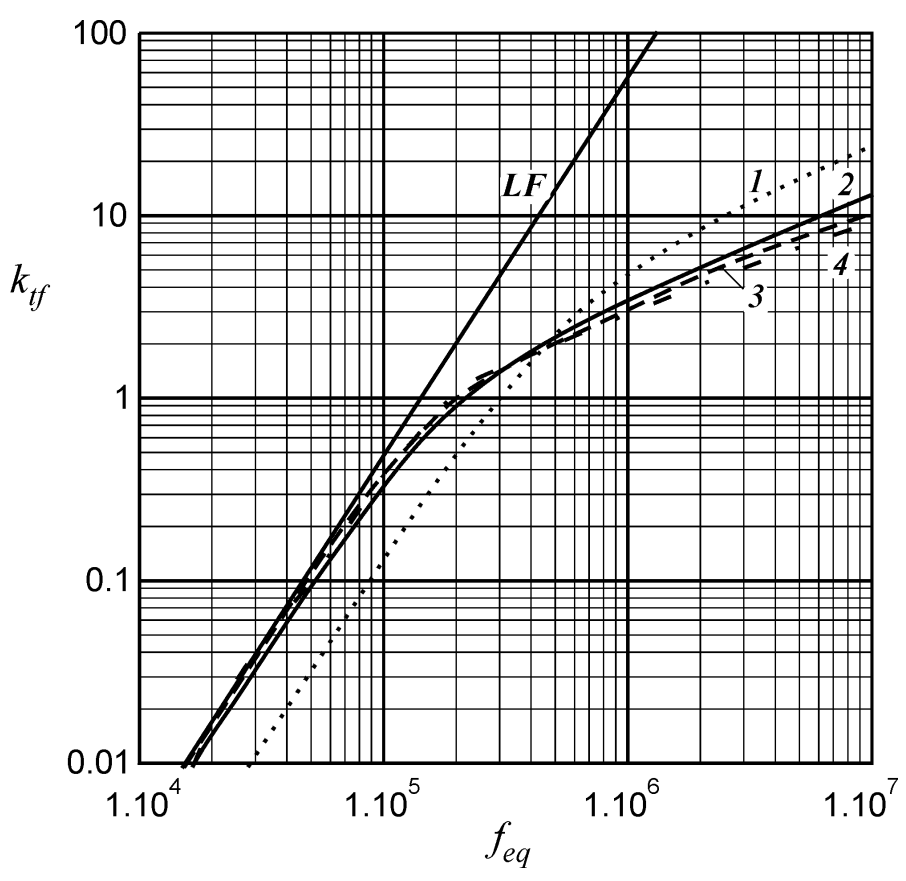

Fig. 2 Transformer cases, typical transformer factor $k_{t f}$ for $\mathrm{d}=0.5 \mathrm{~mm}, \eta=0.7$, $\rho=23 \times 10^{-9}$ and $\left.\lambda=0.5,1\right)$ dotted line: half layer, $\left.m_{E}=0.5 ; 2\right)$ solid line: single layer, $m_{E}=1 ; 3$ ) dashed: two layers, $\left.m_{E}=2 ; 4\right)$ dash-dot: three or more layers, $m_{E}>2$. $L F-$ low frequency approximation.

To unify the use of the graphs, we introduce a reference diameter, an apparent frequency and an equivalent frequency. 


\section{B. Apparent frequency calculation}

In (2), the apparent frequency should be used.

1. In general, one has to sum the contribution of each current harmonic in order to calculate losses. In the presented method, for sinusoidal currents no corrections have to be made and the apparent frequency $f_{a p}$ is the real one:

$$
f_{a p}=f
$$

2. For a symmetrical triangular current waveform, in the low frequency approximation we obtain the following apparent frequency $f_{a p}$ :

$$
f_{a p}=f \frac{2 \sqrt{3}}{\pi} \approx 1.10 f
$$

3. At high frequency, the losses tend to increase with the root of frequency for a given current. In that case, the contribution of harmonics in the current is low and one can use the rms value of the current instead of summing over all harmonics. For a symmetrical triangular current waveform, this results in an apparent frequency $f_{a p}$ :

$$
f_{a p} \approx 1.025 f
$$

\section{Reference wire diameter}

The choice of $0.5 \mathrm{~mm}$ as a reference wire diameter is done in order to use a typical wire diameter for power electronics. The frequency, for which the penetration depth is equal to the reference diameter $d=\delta$, is $20 \mathrm{kHz}$. The limit of the 'low frequency (LF) approximation' for the reference diameter $d=0.5 \mathrm{~mm}$ is $50 \mathrm{kHz}$, thus LF can be applied below $50 \mathrm{kHz}$ for that wire diameter. These values are easy to remember. The diameters of wires in adjacent layers are taken equal and in a square fitting. This is the worst-case design, as a hexagonal fitting usually reduces the losses.

\section{Equivalent frequency calculation}

To use the provided graphs (Fig.1, Fig.2) for any frequency, wire diameter and conductor resistivity, the equivalent frequency of the considered case should be first found:

$$
f_{e q}=f_{a p}\left(\frac{d}{0.5}\right)^{2}\left(\frac{23 \times 10^{-9}}{\rho_{c}}\right)
$$

where:

$f_{a p}$ is the apparent frequency;

$d$ is the wire diameter in [mm];

$\rho_{c}$ is the conductor resistivity in $[\Omega \mathrm{m}]$.

For fast calculation, the waveforms and resistivity could be neglected, but the diameter effect has still to be taken into account by the following simplified expression:

$$
f_{e q} \approx f\left(\frac{d}{0.5 \mathrm{~mm}}\right)^{2}
$$

\section{SHORT EXAMPLES OF APPLYING THE PROPOSED APPROACH}

The graphs shown in Fig.1 and Fig.2 concern a design example with a typical wire diameter of $0.5 \mathrm{~mm}$ and the usual frequency range for power electronics: $10 \mathrm{kHz}$ to $10 \mathrm{MHz}$. For more than two layers $\left(m_{E}>2\right)$, the result is almost independent of the number of layers. The usual values of $\eta$ in transformers are between 0.7 (typical for thin wires and Litz wire) and 0.9 (typical for $\mathrm{d}>0.5 \mathrm{~mm}$ ). For other values of $\eta$, a linear interpolation between Fig.1 and Fig.2 can be done. The additional error due to that interpolation is below $2 \%$.

A few short examples of transformer designs show the use of the graphs in Fig.1 and Fig.2.

1) A single layer winding of a transformer uses a wire diameter of $0.9 \mathrm{~mm}$ and an outer diameter of $1 \mathrm{~mm}$, the frequency is $30 \mathrm{kHz}$, the copper resistivity is $\rho=23 \times 10^{-9} \Omega \mathrm{m}$.

We have $\eta=0.9 \mathrm{~mm} / 1 \mathrm{~mm}=0.9$, so we use Fig. 1 . We have to keep the same diameter/penetration depth ratio, i.e. to find the equivalent frequency $f_{e q}=30 \mathrm{kHz} \times(0.9 / 0.5)^{2}=97.2 \mathrm{kHz}$.

Reading from Fig. 1 gives $k_{t f}=0.473$. It is a single layer transformer, so $m_{E}=1$ and we obtain $k_{c}=k_{t f}=0.473$.

2) The same wire diameters and resistivity as in 1), but a three-layer transformer winding, using a three times smaller winding width, the same turn number.

We use the same values $\eta=0.9$ and $f_{e q}=97.2 \mathrm{kHz}$. It is a three-layer transformer, so $m_{E}=3$. For 3 layers and more, we read $k_{t f}=0.575$ from Fig. 1 . Then we have $k_{c}=3^{2} k_{t f}=5.17$.

3) The same wire diameters and resistivity as above, but a half layer transformer design (the considered single layer secondary is sandwiched between two primary windings).

Using the same values $\eta=0.9$ and $f_{e q}=97.2 \mathrm{kHz}$, we read from Fig. $1 k_{t f}=0.166$. It is a half-layer transformer, so $m_{E}=0.5$ and we have $k_{c}=0.5^{2} k_{t f}=0.0415$. This value is much lower than the cases 1) and 2) above. The reason is that in this design case, the transverse field is zero and only local fields are present. In practical realizations, the half layer solutions indeed perform well, but a non-careful winding (e.g. non-equal winding width of the layers) generates parasitic transverse fields that increase the losses considerably.

4) A two layer transformer winding of $0.5 \mathrm{~mm}$ copper diameter at $50 \mathrm{kHz}$ and packing factor in the direction of the layer $\eta=0.8$, copper resistivity $\rho_{\mathrm{c}}=23 \times 10^{-9} \Omega \mathrm{m}$.

We have the same diameter as the graphs, so the equivalent frequency is equal to the applied frequency. We obtain $k_{t f, 0.9}=0.170$ from Fig. 1 and $k_{t f, 0.7}=0.104$ form Fig.2. To find $k_{t, 0.8}$ (for $\eta=0.8$ ), we take the average of the two values $k_{t f, 0.8}=(0.104+0.170) / 2=0.137$. Then we find $k_{c}=2^{2} k_{t f, 0.8}=0.549$. 


\section{PRACTICAl IMPLEMENTATION OF THE PROPOSED APPROACH}

\section{A. Realized Transformer Design}

A welding transformer is designed using the "fast design approach", proposed in [12]. The method, presented here, is applied for calculating the eddy current losses of the primary and secondary windings of that transformer.

The transformer contains 2 identical coils connected in parallel. Each coil contains a primary winding with number of turns $\mathrm{N}=20$. Each primary winding includes two windings in parallel, Fig.3. Secondary winding of each coil (5 turns, 2 conductors in parallel) is sandwiched between the two primary windings of each coil.

The arrangement of the windings is shown in Fig.3.

The realized transformer is pictured in Fig.4.

The specifications of the designed transformer are:

$\mathrm{U}_{\text {pri }}=300 \mathrm{~V} ; \mathrm{P}_{\text {out }}=2.5 \mathrm{~kW}$ (continuous power)

Core: U 80/40/25; Primary Winding: $\mathrm{N}=20, \mathrm{~d}=1.18$ [mm]; Secondary Winding: $\mathrm{N}=5, \mathrm{~d}=2[\mathrm{~mm}]$

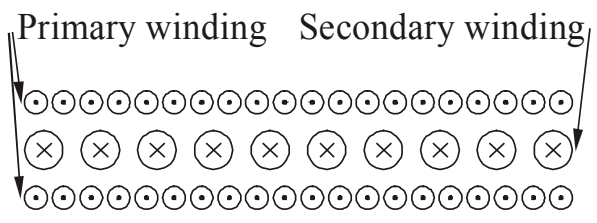

Fig. 3. Designed welding transformer - winding arrangement.

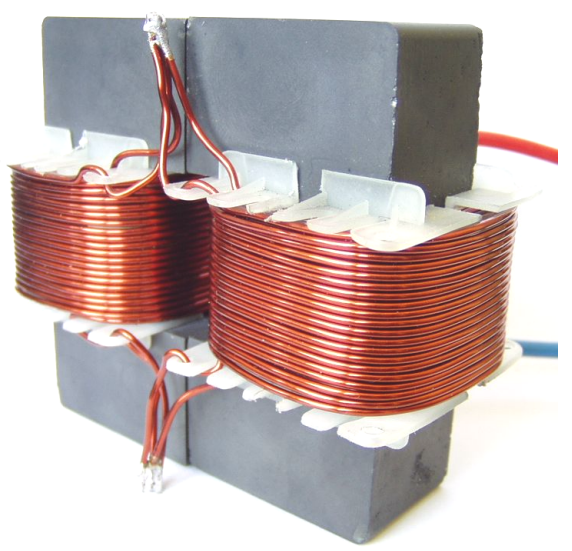

Fig. 4. Realized welding transformer, UU core, N87 ferrite.

\section{B. $\quad$ Winding Loss Calculations}

Primary Winding -A single layer winding, wire diameter of $1.18 \mathrm{~mm}$, the frequency is $60 \mathrm{kHz}$, the copper resistivity is $\rho=21 \times 10^{-9} \Omega \mathrm{m}$.

We have $\eta=d N p / w=1.18 m m \times 20 \times 1 / 26 \mathrm{~mm}=0.908$. We have to keep the same diameter/penetration depth ratio, i.e. we have to find the equivalent frequency. We use (7)

$$
f_{e q, 1}=60 \mathrm{kHz}\left(\frac{1.18}{0.5}\right)^{2}\left(\frac{23}{21}\right)=366 \mathrm{kHz}
$$

We read $k_{t f, 0.9}=2.15$ from Fig. 1 for $\eta=0.9$. The actual value $\eta_{2}=0.908$ is very close to $\eta=0.9$. It is a single layer transformer, so $m_{E}=1$ and we obtain $k_{c}=k_{t f}=2.15$.

Then, using (2) we obtain the eddy current losses of the primary winding. The total losses in the primary winding are the sum of ohmic and eddy current losses.

Secondary Winding - A half layer transformer design $m_{E}=0.5$ (the considered single layer secondary is sandwiched between two primaries), 2 wires in parallel, wire diameter of 2 $\mathrm{mm}$, the frequency is $60 \mathrm{kHz}$, the copper resistivity is $\rho$ $=21 \times 10^{-9} \Omega \mathrm{m}$.

We have $\eta=d N p / w=2 m m \times 5 \times 2 / 26 \mathrm{~mm}=0.769$. The equivalent frequency is

$$
f_{e q, 1}=60 \mathrm{kHz}\left(\frac{2}{0.5}\right)^{2}\left(\frac{23}{21}\right)=1.051 \mathrm{MHz}
$$

We find $k_{t f, 0.9}=6$ from Fig. 1 for $\eta=0.9$ and $k_{t f, 0.7}=4.7$ form Fig. 2 for $\eta=0.7$. The actual value of $\eta$ for the secondary is $\eta_{2}=0.769$. Using the found two values and interpolating for $\eta_{2}=0.769$, we obtain $k_{t f} \approx 5.149$. It is a half layer transformer, so $m_{E}=0.5$ and we obtain $k_{c}=0.5^{2} k_{t f}=1.287$.

The calculated results for the total losses of both primary and secondary windings are tabulated in Table 1.

TABLE 1 CALCULATED RESULTS FOR THE EXPERIMENTAL TRANSFORMER

\begin{tabular}{|c|c|c|c|c|c|}
\hline & $k_{t f}$ & $k_{c}$ & $P_{o h m}[\mathrm{~W}]$ & $P_{e d d y}[\mathrm{~W}]$ & $P_{c u, i}[\mathrm{~W}]$ \\
\hline primary & 2.15 & 2.15 & 1.455 & 3.129 & 4.584 \\
\hline secondary & 5.149 & 1.287 & 2.059 & 2.65 & 4.709 \\
\hline
\end{tabular}

The total losses in primary and secondary windings are:

$$
P_{c u}=P_{c u, 1}+P_{c u, 2}=4.584+4.709=9.293
$$

\section{Measured Winding Losses}

We measured the winding losses $P_{\text {meas }}$ of the realized transformer (short circuit test, primary current 10A) and the results are shown in Table 2, together with the calculated results $P_{\text {total,cu}}$.

TABLE 2 MEASURED WINDING LOSSES $P_{\text {MEAS }}$ UNDER SHORT CIRCUIT TEST, 10 A

\begin{tabular}{|c|c|c|c|}
\hline Measured results & \multicolumn{3}{|c|}{ Calculated results } \\
\hline$P_{\text {meas }}[\mathrm{W}]$ & $P_{c u}[\mathrm{~W}]$ & $P_{\text {end }}[\mathrm{W}]$ & $P_{\text {total,cu }}[\mathrm{W}]$ \\
\hline 9.31 & 9.293 & 0.11 & 9.403 \\
\hline
\end{tabular}

Remark: In Table $2 P_{\text {end }}$ are the losses due to the ends of the secondary winding.

The calculated values for the total copper losses $\mathrm{P}_{\text {total }}=9.403 \mathrm{~W}$ are very close to the measured values $\mathrm{P}_{\text {meas }}=9.31 \mathrm{~W}$. Nevertheless, we expect a general accuracy of $3 \%$ on eddy current losses. The tolerances on dimensions of the wires and other dimensions can also affect the practical accuracy as well. 


\section{CONCLUSION}

The advantages of the proposed approach are the fast and straight calculation combined with good accuracy and wide application. The method is applicable for a wide variety of transformers with different frequencies, wire diameters and conductor arrangements.

The proposed method is used in designing several welding transformers. The target power of the transformers is $2.5 \mathrm{~kW}$. The experiments show good matching with the calculations.

\section{ACKNOWLEDGMENT}

The paper was developed in the frames of the NATO Research Program, Project RIG.981.482.

\section{REFERENCES}

[1] P.L. Dowell, "Effects of eddy currents in transformer windings", Proc. Inst. Elect. Eng., vol. 113, No.8, August 1966, pp.1387-1394.

[2] P.N. Murgatroyd, 'Calculation of proximity losses in multistrand conductor bunches', IEE Proceedings - B, 1992, vol.139, No 1, pp. 2128.

[3] C.R. Sullivan, "Computationally Efficient Winding Loss Calculation with Multiple Windings, Arbitrary Waveforms, and Two-Dimensional or Three-Dimensional Field Geometry", IEEE Transactions on Power Electronics, vol. 16, No 4, January 2001, pp. 142-150.

[4] P. Wallmeier, N. Frohleke, H. Grotstollen, "Improved analytical modeling of conductive losses in gapped high-frequency inductors", IEEE-IAS Annual Meeting, 1998, pp. 913-920.
[5] C.R. Sullivan, "Winding loss calculation with multiple windings, arbitrary waveforms and 2-D field geometry', IEEE IAS Annual Meeting, 1999, pp. 2093-2099.

[4] W.G. Hurley, E. Gath and J. G. Breslin, "Optimized Transformer Design: Inclusive of High-Frequency Effects", IEEE Transactions on Power Electronics, vol. 13, No 4, Yuly 1998, pp. 651-658.

[5] W.G. Hurley, E. Gath and J. G. Breslin, "Optimising the AC Resistance of Multilayer Transformer Windings with Arbitrary Current Waveforms", IEEE Transactions on Power Electronics, vol. 15, No 2, March 2000, pp. 369376.

[6] R. Petkov, "Optimum Design of a High-Power, High-Frequency Transformer", IEEE Transactions on Power Electronics, vol. 11, No 1, January 1996, pp. 33-42.

[7] J. Ferreira, 'Improved analytical modelling of conductive losses in magnetic components', IEEE Transactions on Power Electronics, vol. 9, No 1, 1994, pp. 127-131.

[8] J. Ferreira, 'Analytical computation of AC resistance of round and rectangular litz wire windings' IEE Proceedings - B, vol. 139, No 1, 1992, pp. 21-25.

[9] C.R. Sullivan, "Cost-constrained selection of strand diameter and number in a litz-wire transformer", IEEE Transactions on Power Electronics, vol. 16, No 2, March 2001, pp. 281-288.

[10] J.D Lavers. and V. Bolborici "Loss Comparison in the Design of High Frequency Inductors and Transformers", IEEE Transactions on Magnetics, vol. 35, No 5, September 1999, pp. 3541-3542.

[11] Alex Van den Bossche, Vencislav Valchev, 'Inductors and Transformers for Power Electronics', , CRC Press, Boca Raton, Florida, USA. 2005, 480 pp.

[12] V.C. Valchev, A.Van den Bossche, 'Design method for power electronic magnetic components including eddy current losses - Part 2', $1^{\text {st }}$ International Congress, MEET-MARIND, Varna, Bulgaria, 7-11 October, 2002, pp. 317-325. 